\title{
Comparative Analysis of Methods for Enhancement of the Photostability of CdTe@TGA QDs' Colloid
}

\section{Solutions}

Aleksey S. Tsipotan,,$^{\dagger}$ Marina A. Gerasimova, ${ }^{\dagger}$ Sergey P. Polyutov, ${ }^{\dagger}$ Aleksandr S. Aleksandrovsky, ${ }^{\prime *}$ Vitaliy V. Slabko ${ }^{*}$

${ }^{\dagger}$ Siberian Federal University, Krasnoyarsk, 660041, Russia

${ }^{\ddagger}$ Kirensky Institute of Physics, Russian Academy of Sciences, Krasnoyarsk, 660036, Russia

ABSTRACT: The employment of colloid quantum dots in a number of applications is limited by their instability under light irradiation. Additional methods of photostability enhancement of UV+visible-irradiated TGA-stabilized CdTe quantum dots are investigated. Photostability enhancement was observed via either addition of sodium sulphite in the role of chemical oxygen absorber or addition of $1 \%$ of gelatin, or, finally, by additional stabilization by bovine serum albumine (BSA). The latter method is the most promising since it not only enhances the quantum dots' photostability but also makes them more biocompatible and extends the possibilities of their biological applications. 


\section{INTRODUCTION}

Semiconductor nanocrystals (colloid quantum dots (QD)) attract great attention in recent years due to numerous possible applications, e.g., in life science, ${ }^{1-3}$ in sensorics, ${ }^{4-6}$ in display technology ${ }^{7}$ as LEDs, ${ }^{8,9}$ solar energy harvesting. ${ }^{10-12}$ The progress in these studies is sometimes hindered by the limitations of chemical stability and photostability of QDs in solution. ${ }^{13}$ The latter is especially important in the physical regimes when QDs undergo long-lasting or multiply repeated optical irradiation. Such the regimes occur, for example, when QDs are utilized for cellular labeling and biological imaging, ${ }^{14,15}$ or in the processes of self-assembly of QDs into the structures of pre-defined geometry in the field of laser radiation. ${ }^{16}$ The role of dissolved oxygen in the photoinduced instability of colloidal QDs is well known. ${ }^{14}$ Ma et al argued that optical irradiation stimulates generation of reactable state of oxygen, with the sequent oxidation of both stabilizer and the surface of QDs, and final destruction or aggregation of QDs. Enhancement of QDs' solutions photostability is known to be attained either by chemical extraction of oxygen from the solution or by additional stabilization of QDs via preventing oxygen access to the QDs. Serum Albumin (BSA) is well studied and widely used protein which enables modification of nanoparticles surfaces with the size of several tens of $n m{ }^{17,18}$ or for their binding. ${ }^{19}$ Employing of BSA as the additional surface passivation agent allows obtaining stable and biocompatible QDs with improved luminescent properties. ${ }^{20}$ Interaction of QDs with BSA leading to their mutual binding ${ }^{21-26}$ as well as their $\mathrm{pH}$ stability ${ }^{27,20}$ are well documented. On other side, the photostability of several BSA-QD complexes was surprisingly flabbily studied. Let us mention just a few. Improvement of QDs photostability in 10\% gelatin solution under $400 \mathrm{~nm}$ irradiation was demonstrated in paper. ${ }^{28}$ Improvement of BSA-QD photostability under 405 and $800 \mathrm{~nm}$ 
irradiation was evidenced. ${ }^{29}$ Let us also mention that regardless of well known fact that photooxidation, degradation and aggregation of QDs are most pronounced under UV irradiation ${ }^{13,30}$ here are no any sufficient studies in the literature on the additional stabilization efficiency under UV irradiation. The purpose of the present study is the investigation of photostability improvement of QDs under UV irradiation using all known approaches, namely, chemical oxygen extraction, viscosity modification of the solution by gelatin and additional stabilization with BSA.

\section{EXPERIMENTAL SECTION}

Materials and Sample Preparation. Water-soluble CdTe QDs under study were produced by PlasmaChem and then were stabilized by thioglicole acid (TGA). All solutions were prepared using the phosphate buffer $(0.02 \mathrm{M})$ with $\mathrm{pH}=6.9$. Reference sample containing only TGAstabilized QDs was prepared by mixing $1960 \mu 1$ of buffer solution and $40 \mu 1$ of QDs solution (C $\left.=3 \cdot 10^{-4} \mathrm{M}\right)$. The sample for a chemical oxygen absorber test was prepared using the buffer solution $(1940 \mu \mathrm{l}), \mathrm{Na}_{2} \mathrm{SO}_{3}\left(20 \mu \mathrm{l}\right.$ of $1 \mathrm{M}$ solution) and $40 \mu \mathrm{l}$ of QDs' solution $\left(\mathrm{C}=3 \cdot 10^{-4} \mathrm{M}\right)$. Fraction of sodium sulphite content in this sample was chosen to be ten times higher than that necessary to remove all dissolved oxygen. The $1 \%$ gelatin (Fluka) solution was prepared by dissolving $52.7 \mathrm{mg}$ of dry gelatin in $5.27 \mathrm{ml}$ of buffer under continuous stirring in a magnetic stirrer and $80^{\circ} \mathrm{C}$ heating. The sample for gelatin efficiency test was prepared of $1960 \mu 1$ of $1 \%$ gelatin solution and $40 \mu \mathrm{l}$ CdTe solution $\left(\mathrm{C}=3 \cdot 10^{-4} \mathrm{M}\right)$. BSA solution $\left(\mathrm{C}=5 \cdot 10^{-4} \mathrm{M}\right)$ was prepared just right before the BSA efficiency test experiment using of $5.3 \mathrm{mg}$ of dry BSA and 
$160.6 \mu 1$ of phosphate buffer. The sample for BSA efficiency test was prepared of $1910 \mu 1$ of buffer, $50 \mu \mathrm{l}$ of BSA solution $\left(\mathrm{C}=5 \cdot 10^{-4} \mathrm{M}\right)$, and $40 \mu \mathrm{l}$ of $\mathrm{CdTe}$ solution $\left(\mathrm{C}=3 \cdot 10^{-4} \mathrm{M}\right)$.

Instrumentation. Absorption spectra were recorded in a quartz cuvette $(10 \times 10 \mathrm{~mm})$ using Lambda 35 UV-vis spectrophotometer (Perkin-Elmer). PL spectra were excited at $480 \mathrm{~nm}$ and recorded using Fluorolog 3 spectrofluorometer (HORIBA JobinYvon) with excitation and emission slit widths $1.2 \mathrm{~nm}$. All measurements were performed at room temperature. Colloid solutions were illuminated using DRSh -250-3M mercury lamp. Irradiance spectrum of this lamp is plotted in Figure 1 and contains a number of intense lines in the range from 275 to $600 \mathrm{~nm}$. Power of radiation over the aperture of colloid solution was measured by thermopile light sensor and was found to be $\mathrm{P}=0.067 \mathrm{~W}$.

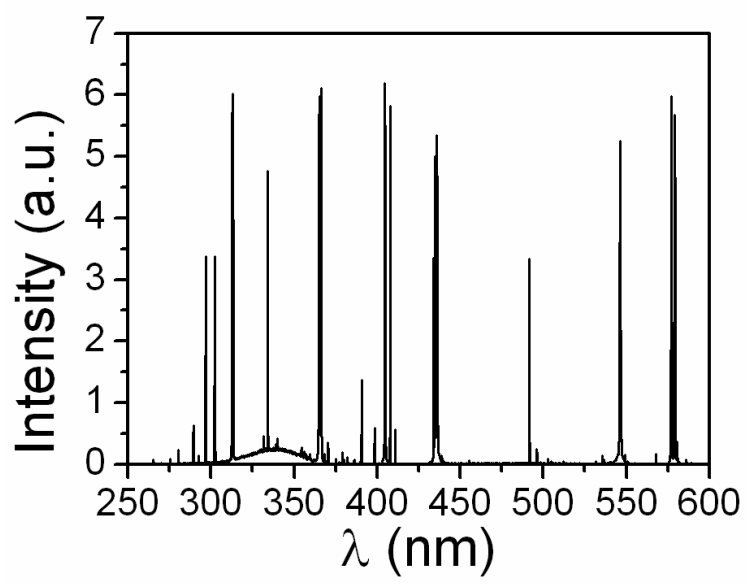

Figure 1. Spectra of DRSh -250-3M mercury lamp used for CdTe QDs' solution illumination.

RESULTS AND DISCUSSION

Absorption and photoluminescence spectra. Absorption and photoluminescence spectra of the samples before irradiation are shown in Figure 2. Luminescence of CdTe QDs in 1\% gelatin 
solution is approximately $50 \%$ higher than that of reference sample (QDs in pure buffer solution), and luminescence band peak wavelength experiences no any shift, while absorption remains almost the same, compare both with the position and amplitude of CdTe QDs exciton peak. Luminescence of BSA-stabilized QDs solution is about 25\% higher than that of reference sample, while luminescence peak experiences $5 \mathrm{~nm}$ blue shift. Again, absorption in this case experiences no any changes. Impurity of $\mathrm{Na}_{2} \mathrm{SO}_{3}$ as the oxygen absorber does not change optical properties of QDs' solution.

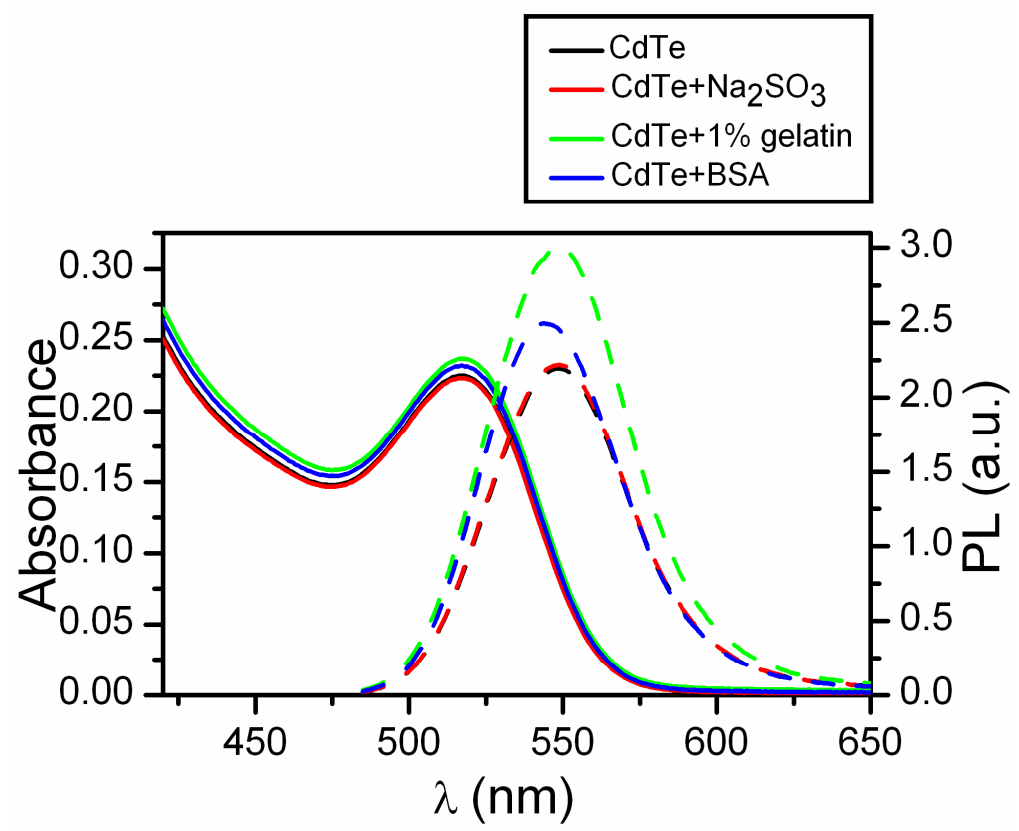

Figure 2. Optical density of $0.9 \mathrm{~cm}$ thick CdTe solution (solid line) and photoluminescence spectra (dash line).

QDs' solutions were irradiated for 165 min (reference sample and $\mathrm{Na}_{3} \mathrm{SO}_{3}$-containing sample) or $335 \mathrm{~min}$ (gelatin-stabilized and BSA-stabilized samples). Photostability was examined repeatedly, by recording absorption and photoluminescence spectra after each 15 min period of irradiation. 
Figure 3 shows recorded absorption spectra of the samples. One can see that QDs in the buffer solution exhibit weak photostability (Figure 3a and Figure 4a), since optical density at the wavelength of the exciton peak and luminescence peak drops during first 15 minutes of irradiation by $5 \%$ and $20 \%$, correspondingly. Absorption experiences $19 \%$ drop after first 60 min of irradiation. Next 105 min of irradiation results in decreasing of these values by $60 \%$ and $91 \%$, correspondingly. Simultaneously, optical density at the wavelengths larger than exciton absorption peak wavelength of isolated QD increases. Both of these facts origin either due to variation of quantum confinement effect or due to QDs aggregation. As it was already discussed above all current studies of photodegradation or any other kind of degradation of QDs' solutions imply key role of oxygen. Photochemical reaction of oxygen with the stabilizer results in gradual loss of anti-aggregation stability and subsequent aggregation. Photochemical reaction of oxygen with the surface of QD leads to decreasing of QD's size resulting in blue shift of exciton peak, as clearly seen in Figure 3a. 

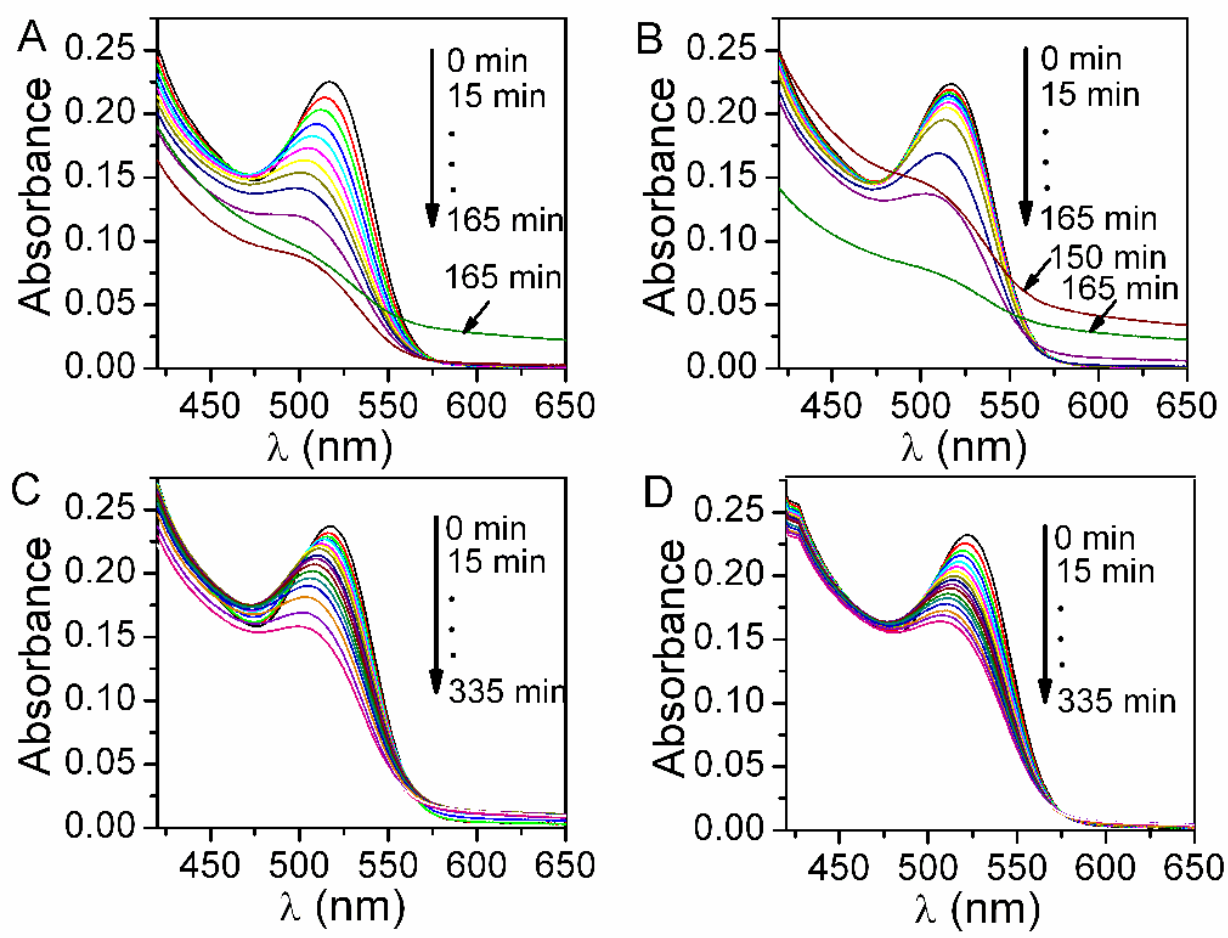

Figure 3. Variation of absorption spectra of QDs' solutions in the course of irradiation. (a) CdTe in the buffer, (b) CdTe in the presence of $\mathrm{Na}_{2} \mathrm{SO}_{3}$, (c) CdTe in $1 \%$ gelatin solution, (d) CdTe stabilized by BSA. 

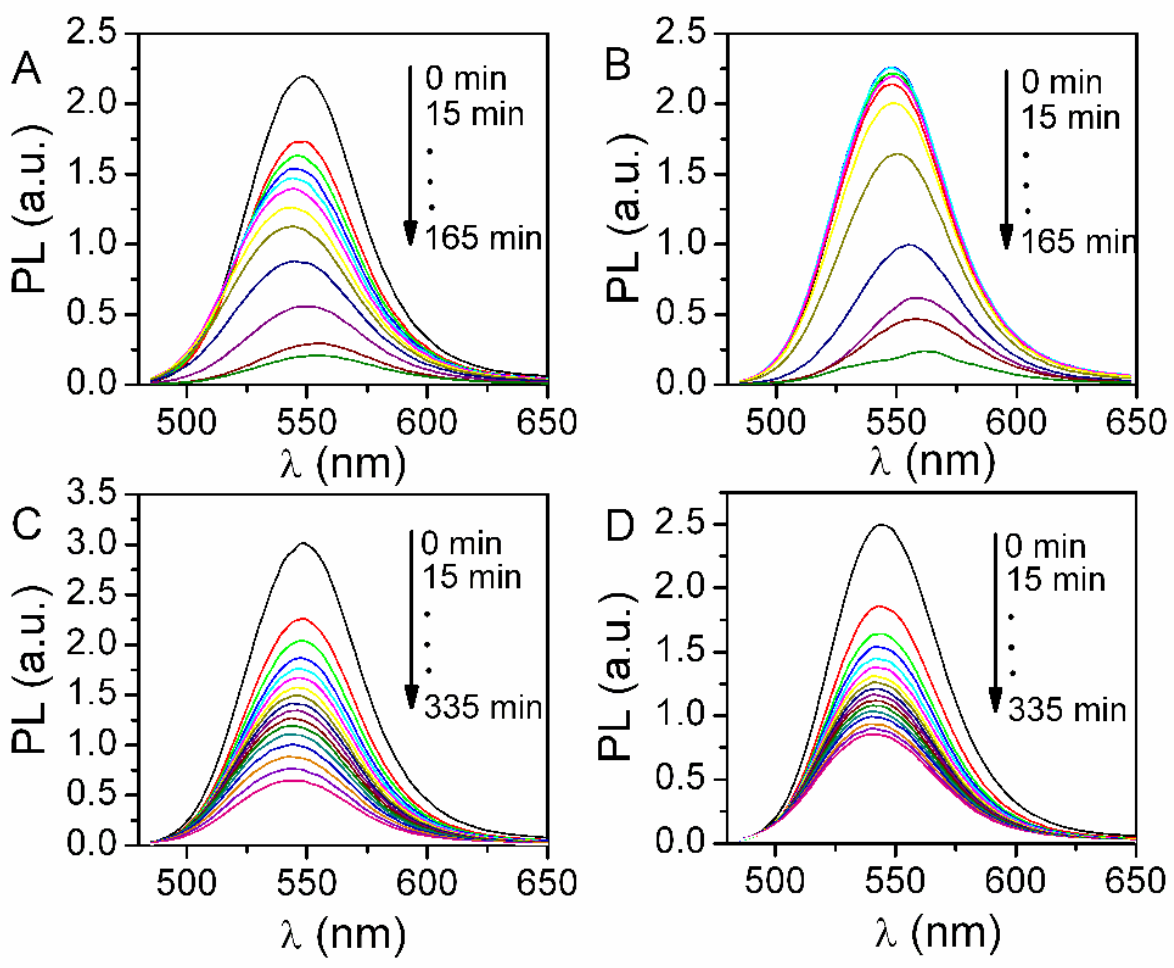

Figure 4. Variation of photoluminescence spectra of solutions. (a) CdTe in a buffer solution (b) CdTe with $\mathrm{Na}_{2} \mathrm{SO}_{3}$, (c) CdTe in 1\% gelatin solution, (d) CdTe stabilized by BSA.

Impurity of sodium sulfite (NSO) to QDs solution results in reduction of oxygen centent fraction in the sample. As a sequence, the photostability of corresponding solution becomes higher and solution behavior under irradiation is noticeably modified. Drop of optical density of NSO-stabilized QDs (Figure 3b) after the first hour of illumination is about 5\% while that of reference solution is $19 \%$. However, during the second hour of illumination, the drop of optical density becomes more and more pronounced achieving 60\% after $135 \mathrm{~min}$. One can see in Figure $3 \mathrm{~b}$ that at time $150 \mathrm{~min}$ (red line) optical density increases compare to the one at $135 \mathrm{~min}$ (violet line). This effect is connected with increased scattering of light in the spectrometer during 
initial stage of aggregation. Hence, the addition of NSO leads to increase of QDs' photostability during an hour of irradiation of used power $(\mathrm{P}=0.067 \mathrm{~W})$, while at larger irradiation times this effect gradually vanishes. Luminescence intensity, in contrast, exhibits minor increase of $2 \%$ after first 75 min of illumination, followed by sharp drop in the range $70-120$ minutes, resulting in $90 \%$ loss of luminescence after 165 min of irradiation, and finally results in termination of stabilization by NSO. This behavior of luminescence correlates in time with the initial stage of aggregation. However, both absorption and luminescence evolution for NSO-stabilized sample within 165 min period is not as gradual as in case of reference sample. The latter can be connected with two processes occurring in the solution containing $\mathrm{Na}_{2} \mathrm{SO}_{3}$. As it was shown in a number of studies, ${ }^{31,32} \mathrm{CdSe}$ quantum dots dissolved in water with impurity of sodium sulphite can be used for production of hydrogen by irradiation of the solution. In this case sodium sulphite acts as the donor of electrons, ${ }^{31,}{ }^{32}$ which recombine with photoinduced holes in the valence band of QDs, and cadmium serves as electron acceptor. Since electron acceptor is absent in our experiments, irradiation of solution must lead to charging of QDs and emerging of situation which is analogous to A-type blinking of a solitary QD. ${ }^{33}$ Herewith, the process of hydrogen production starts after a certain delay ${ }^{32}$ with the duration being determined by initial sodium sulfite fraction in the solution. During that delay, sodium sulfite acts as the oxygen absorber thus preventing QDs from oxidation and preserving the luminescence brightness of QDs. The second process occurring in NSO-stabilized QDs' solution is the transfer of oxygen from the ambience surrounding to a solution due to diffusion and convective flows that occurs due to inhomogeneous heating of the cell by the optical radiation. As a result of two processes described above, sodium sulfite impurity falls to zero during irradiation time. The onset of both 
QDs' oxidation and "blinking" leads to sharp collapse of the QDs luminescence observed in our experiment after 90 min of illumination irradiation.

Gradual introduction of sodium sulfite into the QDs solution during the course of irradiation promises further increase of photostability, though this method can meet limitation in view of the growth of ionic strength of the solution which may influence the QDs' chemical stability.

Since QDs' photodegradation rate depends on the presence of free oxygen in a solution, ${ }^{34,35}$ decrease of its diffusion rate allows reduction of both rate of collisions between QDs and oxygen molecules and the rate of oxygen transfer from ambience to a solution. Addition of, e.g. $1 \%$ of gelatin to the buffer solution is shown ${ }^{36}$ to increase its viscosity by 2.3 , leading to 2.3 -fold decrease of oxygen diffusion rate. In our experiment, ten-fold photoluminescence quenching occurs during 165 min of illumination of CdTe QDs in the buffer solution. Gelatin-stabilized solution exhibits photoluminescence quenching by $84 \%$ during $330 \mathrm{~min}$, that is, approximately 2 times slower, in accordance with expected variation of oxygen diffusion rate.

As pointed above, QDs' solution illumination leads additionally to photodegradation of a stabilizer playing an important role in the formation of QDs' optical properties. Employment of BSA as additional stabilizer of QDs' must lead to stronger protection against oxygen penetration to both QDs' surface and TGA molecules and, as the sequence, to improvement of QDs' photostability. In our experiment QDs stabilized by joint action of TGA and BSA exhibit enhanced stability than those in the solution with $1 \%$ gelatin. Photoluminescence intensity decrease was $70 \%$ of initial value after 6 hour of illumination, while for gelatin-containing solution it was $84 \%$. Mechanism of degradation of TGA+BSA-stabilized QDs must be the denaturation of BSA primarily under UV irradiation. Particularly, decrease of BSA luminescence at $350 \mathrm{~nm}$ under $254 \mathrm{~nm}$ irradiation was reported and treated as the proof of BSA denaturation. ${ }^{37}$ 
In our experiment, the intensity of irradiation at $254 \mathrm{~nm}$ is negligibly small. To check the presence of denaturation process under the illumination with the radiation with the spectrum presented in Figure 2, we examined BSA luminescence behavior under the same conditions like those used for experiments with QDs. We have found that BSA luminescence at $350 \mathrm{~nm}$ decreases by $60 \%$ after 15 min of irradiation (Figure 5). According to Figures $3 d$ and $4 d$, the most pronounced variation of QDs' spectra associated with their photodegradation is observed in this very time period after the start of illumination.

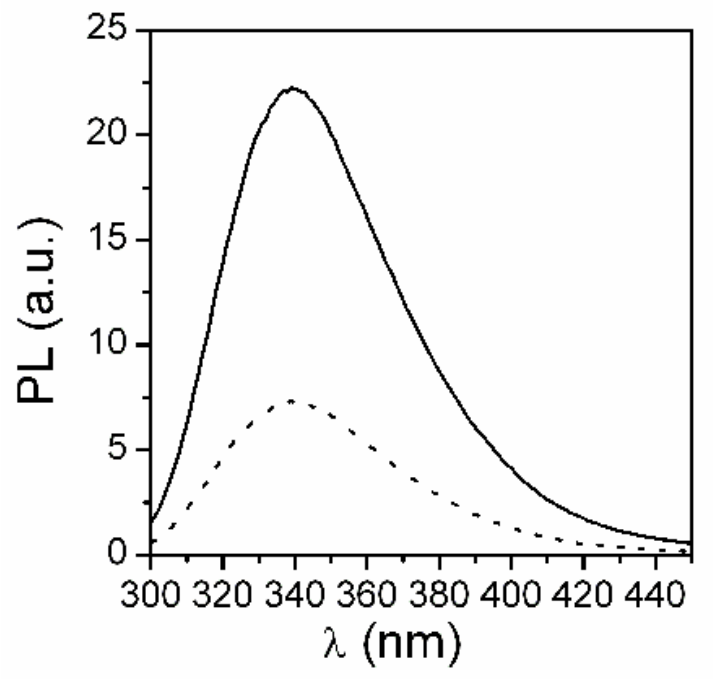

Figure 5. $295 \mathrm{~nm}$ excited luminescence spectra of BSA before irradiation (solid line) and after 15 min irradiation (dashed line).

\section{Variation of spectral maxima for QDs' excitonic absorption and photoluminescence}

under illumination. The spectral shift of QDs' absorption and photoluminescence maxima under the illumination is commonly connected either with the variation of their size ${ }^{13}$ or with their aggregation. ${ }^{38}$ Corresponding data obtained in our experiment are presented in Figure 6. 

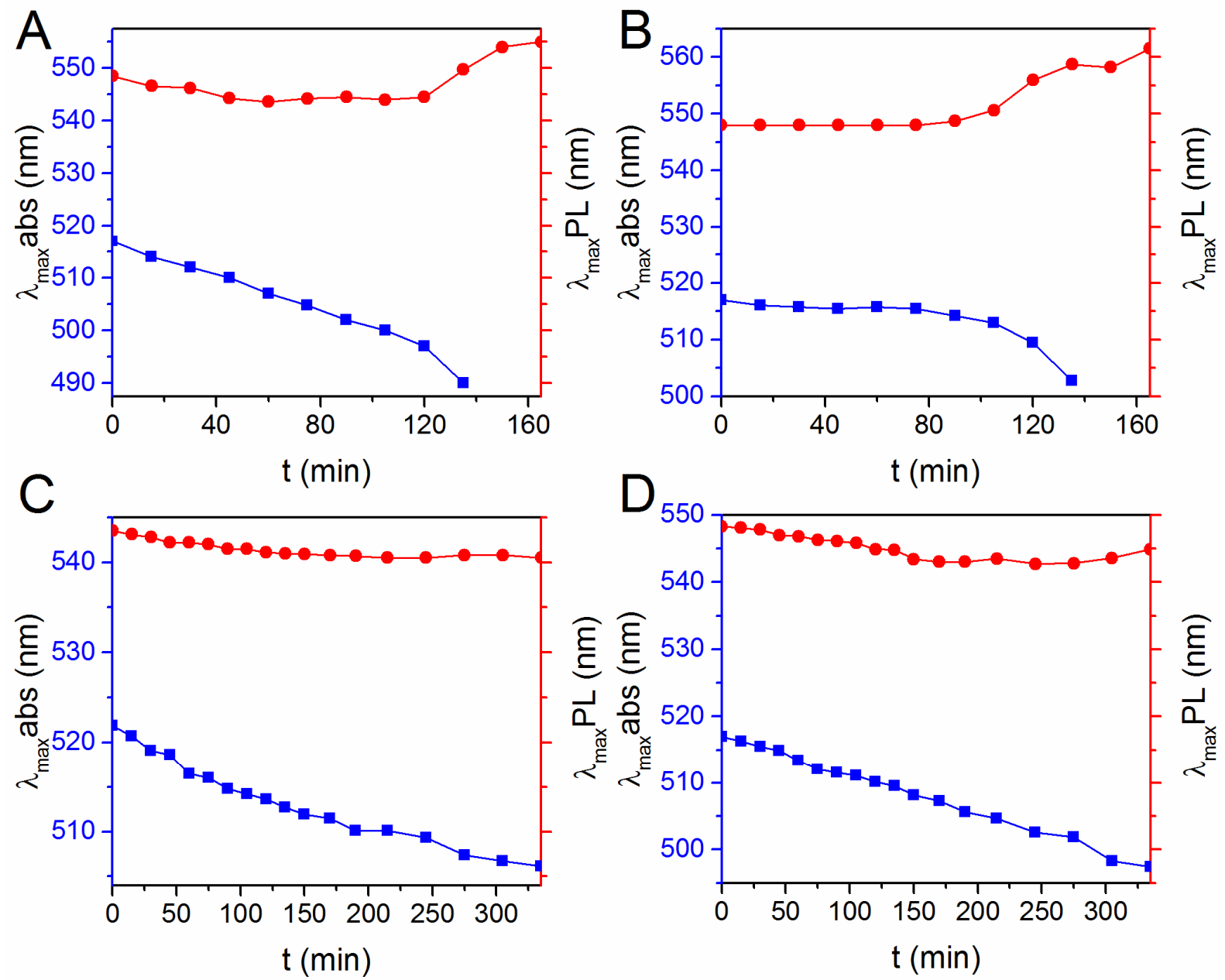

Figure 6. Absorption and photoluminescence maxima shift of QDs in the course of irradiation: (a) $\mathrm{CdTe}$ in the buffer solution (b) CdTe with addition of $\mathrm{Na}_{2} \mathrm{SO}_{3}$, (c) $\mathrm{CdTe}$ in $1 \%$ gelatin solution, (d) CdTe additionally stabilized by BSA.

Blue shift of both absorption and photoluminescence maxima was observed for QDs in the buffer solution (Figure 6a). Note that for irradiation time more than $135 \mathrm{~min}$ the determination of absorption maximum was impossible due to inhomogeneous broadening of the spectrum. This broadening must be explained by the formation of large aggregates and by the electrodynamical interaction of the particles within these aggregates that produces strong modification of spectra. ${ }^{39}$ 
Addition of $\mathrm{Na}_{2} \mathrm{SO}_{3}$ stabilizes both absorption and photoluminescence maxima positions for 90 min of irradiation. At irradiation times more than 1.5 hour maxima begin to shift; however, absorption and photoluminescence maxima experience shift of opposite sign. Specifically, absorption maximum exhibits blue shift till $135 \mathrm{~min}$, while at larger illumination times strong light scattering by massive naked-eye-seen aggregates prevents from determining of absorption maximum position. Photoluminescence maximum experiences red shift evidencing simultaneity of aggregates' formation and reduction of individual QDs' size. Absorption and photoluminescence maxima in gelatin-containing and TGA+BSA-stabilized solutions exhibit just the same behavior. In these two cases the synchronous blue shift of both spectral maxima evidencing the reduction of QDs' size. Loss of transmission due to scattering of light by massive aggregates was not observed both for gelatin-containing and TGA+BSA-stabilized solutions. Therefore, at the examined illumination durations, QDs remain stabilized against aggregation to the sufficient extent.

Kinetics of QDs' photodegradation. The square under the curves describing a band in a photoluminescence spectrum can be treated under certain conditions as the measure of a number of QDs participating in the emission, and therefore, as the measure of QDs' photostability. Figure 7 presents the dependencies of luminescent band's area (S) on the irradiation time as well as their best-fit exponential approximations. 

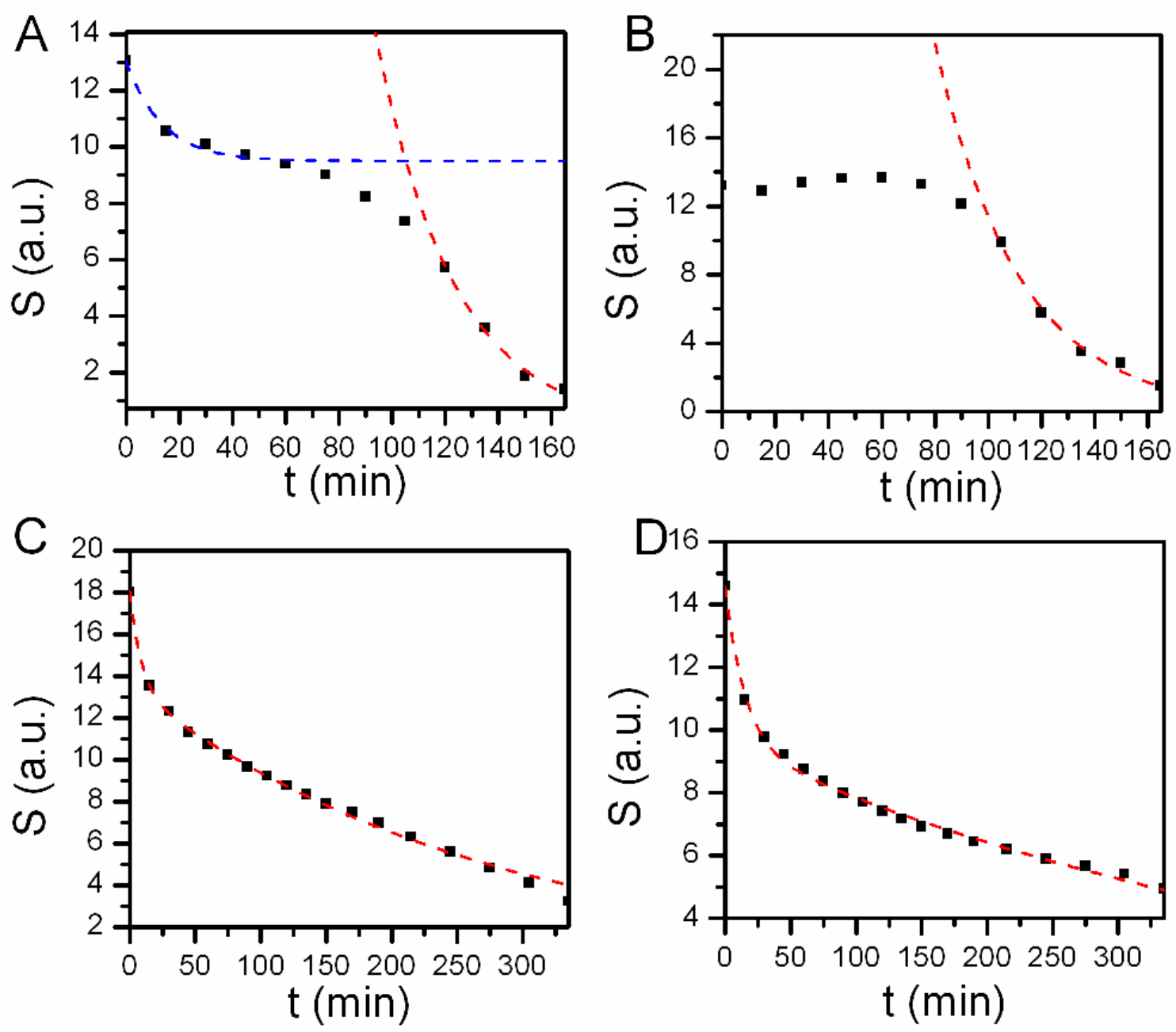

Figure 7. Time dependence of the area under the luminescent band curve (S) for (a) QDs in the buffer solution, (b) QDs in buffer solution with addition of $\mathrm{Na}_{2} \mathrm{SO}_{3}$, (c) QDs with addition of $1 \%$ of gelatin (d) TGA+BSA-stabilized QDs.

The luminescent band area decay for QDs in the buffer solution evidently is a two-stage process with a certain time delay between the first stage and the onset of the second stage (Figure 7a). First stage is governed by the exponent with the time constant of $13 \mathrm{~min}$ and must be ascribed to QDs' surface degradation and QDs' size reduction. First stage is governed by the exponent with the time constant of 13 min and must be ascribed degradation of TGA stabilizing 
layer. Degradation of TGA leads to three consequences overlapping in time, namely, QDs' surface degradation, QDs' size reduction, and gradual loss of stability against aggregation. Decrease of luminescence intensity initially is due to first two factors, while aggregation becomes evident at the second stage, when the density of QDs with degraded TGA layer becomes sufficient. This second stage is governed by the exponent with the time constant $30 \mathrm{~min}$ and becomes completely evident after 120 min of irradiation. The time constant of first stage is determined by the diffusion of oxygen, while the time constant of the second stage is due to QDs' diffusion that is slower due to larger diameter of QDs. The decrease of luminescence at the second stage is due to the sedimentation of large enough aggregates from the solution.

Corresponding dependence for QDs with $\mathrm{Na}_{2} \mathrm{SO}_{3}$ (Figure 7b) contains the time period where luminescent band area can be considered as a constant. The duration of this period is approximately $90 \mathrm{~min}$, and this period must be ascribed to the preserving action of sodium sulfate against the oxygen-mediated photodegradation of QDs. On the expire of $90 \mathrm{~min}$, the decay of luminescent band square is governed by the exponent with $32 \mathrm{~min}$ time constant. This time constant is close to that detected in the pristine buffer solution and must be associated with the onset of aggregation. Decay dependence for gelatin-stabilized solution is described by biexponential law with time constants 9 and $277 \mathrm{~min}$. The same behavior is observed for TGA+BSA-stabilized solution, time constants being 15 and $502 \mathrm{~min}$. The smaller time constant in case of gelatin stabilization can be associated with the action of oxygen dissolved in the close vicinity of every QD wile in case of TGA+BSA it is the time constant of BSA denaturation. Large time constants observed at the second stages of illumination of these two kinds of solutions evidence noticeable enhancement of QDs' photostability when using gelatin or TGA+BSA. 
The photostability of the QDs is extremely urgent for a number of applications. One of the approaches is the coating by other materials such as dielectric ones (e.g. $\mathrm{SiO}_{2}{ }^{40}$ ) or core-shell structuring with semiconductors ${ }^{41}$. Considerable advances were achieved using these approaches. However, the variation of electrical properties of QDs may change the properties of ensembles formed of them, and therefore, to limit the range of possible applications. Probably, the required level of photostability can be achieved by a combination of approaches investigated in the present paper with those employing the additional coating of QDs.

\section{CONCLUSIONS}

Additional enhancement of TGA-stabilized CdTe QDs' photostability under UV irradiation is demonstrated. The methods employed are chemical removal of oxygen, solution viscosity increase, and additional passivation of TGA@QD complexes. Introduction of excessive oxygenbinding $\mathrm{Na}_{2} \mathrm{SO}_{3}$ enables perfect photostability during the time period of order of hours, until the expenditure of this stabilizer. Control of solution viscosity by gelatin addition produces two-fold increase of QDs' photostability. The combined TGA+BSA stabilization of QDs must be considered as the most promising one since it not only enhances the photostability but also makes QDs more biocompatible and extends the possibilities of their applications in biological media.

\section{AUTHOR INFORMATION}

\section{Corresponding Author}

*E-mail: aleksandrovsky@kirensky.ru.

\section{Notes}


The authors declare no competing financial interest.

\section{ACKNOWLEDGMENT}

This research was supported by RFBR and Government of Krasnoyarsk Territory by the research projects No. 16-42-240410r_a, and by Project № 0356-2015-0412 of SB RAS Program №II.2P. VVS is grateful for the support from the Ministry of Education and Science of the Russian Federation (Grant № 3.6341.2017/VU).

\section{REFERENCES}

(1) Yu, L.J.; Seon, K.J.; Chul, P.J.; Sung, N.Y. Protein-quantum Dot Nanohybrids for Bioanalytical Applications. WIREs Nanomed Nanobiotechnol 2016, 8, 178-190.

(2) Kim, T.; Noh, M.; Lee, H.; Joo, S.W.; Lee S.Y.; Lee K. Fluorescence-Based Detection of Point Mutation in DNA Sequences by CdS Quantum Dot Aggregation. J. Phys. Chem. B 2009, 113 (43), 14487-14490.

(3) Priem, B.; Tian, C.; Tang, J.; Zhao, Y.; Mulder W. JM. Fluorescent Nanoparticles for the Accurate Detection of Drug Delivery. Expert Opin. Drug Deliv. 2015, 12(12), 1881-1894.

(4) Ng, S.M.; Koneswaran, M.; Narayanaswamy, R. A Review on Fluorescent Inorganic Nanoparticles for Optical Sensing Applications. RSCAdv. 2016, 6, 21624-21661.

(5) Stanisavljevic, M.; Krizkova, S.; Vaculovicova, M.; Kizek, R.; Adam, V. Quantum DotsFluorescence Resonance Energy Transfer-Based Nanosensors and Their Application. Biosens. Bioelectron. 2015, 74, 562-574. 
(6) Pan, H.; Cui, R.; Zhu, J.J. CdTe Quantum Dots as Probes for Near-Infrared Fluorescence Biosensing Using Biocatalytic Growth of Au Nanoparticles. J. Phys. Chem. B 2008, 112 (51), $16895-16901$.

(7) Kim, T.H.; Cho, K.S.; Lee, E.K.; Lee, S.J.; Chae, J.; Kim, J.W.; Kim, D.H.; Kwon, J.Y.; Amaratunga, G.; Lee, S.Y. et al Full-Colour Quantum Dot Displays Fabricated by Transfer Printing. Nat. Photon. 2011, 5, 176-182.

(8) Yang, Y.; Zheng, Y.; Cao, W.; Titov, A.; Hyvonen, J.; Manders, J.R.; Xue, J.; Holloway, P.H.; Qian, L. High-Efficiency Light-Emitting Devices Based on Quantum Dots with Tailored Nanostructures. Nat. Photon. 2015, 9, 259-266.

(9) Wang, D.Y.; Wang, I.S.; Huang, I.S.; Yeh, Y.C.; Li, S.S.; Tu, K.H.; Chen, C.C.; Chen, C.W. Quantum Dot Light-Emitting Diode Using Solution-Processable Graphene Oxide as the Anode Interfacial Layer. J. Phys. Chem. C 2012, 116 (18), 10181-10185.

(10) Zhao, D.; Yang, C.F. Recent Advances in the TiO2/CdS Nanocomposite Used for Photocatalytic Hydrogen Production and Quantum-Dot-Sensitized Solar Cells. Renew Sust Energ Rev 2016, 54, 1048-1059.

(11) Kamat, P.V. Quantum Dot Solar Cells. Semiconductor Nanocrystals as Light Harvesters. J. Phys. Chem. C 2008, 112 (48), 18737-18753.

(12) Jumabekov, A.N.; Deschler, F.; Böhm, D.; Peter, L.M.; Feldmann, J.; Bein, T. QuantumDot-Sensitized Solar Cells with Water-Soluble and Air-Stable PbS Quantum Dots. J. Phys. Chem. C 2014, 118 (10), 5142-5149. 
(13) Aldana, J.; Wang, Y.A.; Peng, X. Photochemical Instability of CdSe Nanocrystals Coated by Hydrophilic Thiols. J. Am. Chem. Soc. 2001, 123 (36), 8844-8850.

(14) Ma, J.; Chen, J.Y.; Zhang, Y.; Wang, P.N.; Guo, J.; Yang, W.L.; Wang, C.C. Photochemical Instability of Thiol-Capped CdTe Quantum Dots in Aqueous Solution and Living Cells: Process and Mechanism. J. Phys. Chem. B 2007, 111, 12012-12016.

(15) Wang, T; Jiang, X. Size-Dependent Stability of Water-Solubilized CdTe Quantum Dots and Their Uptake Mechanism by Live HeLa Cells. ACS Appl. Mater. Interfaces 2013, 5 (4), 1190-1196.

(16) Tsipotan, A.S.; Gerasimova, M.A.; Slabko, V.V.; Aleksandrovsky, A.S. Laser-Induced Wavelength-Controlled Self-Assembly of Colloidal Quasi-Resonant Quantum Dots. Opt. Express 2016, 24(10), 11145-11150.

(17) Li, Z.; Huang, H.; Tang, S.; Li, Y.; Yu, X.F.; Wang, H.; Li, P.; Sun, Z.; Zhang, H.; Liu, C. et al Small Gold Nanorods Laden Macrophages for Enhanced Tumor Coverage in Photothermal Therapy. Biomaterials 2016, 74, 144-154.

(18) Nooney, R.I.; White, A.; O’Mahony, C.; O’Connell, C.; Kelleher, S.M.; Daniels, S.; McDonagh, C. Investigating the Colloidal Stability of Fluorescent Silica Nanoparticles under Isotonic Conditions for Biomedical Applications. J. Colloid Interface Sci. 2015, 456, 50-58.

(19) Chu, D.; Gao, J.; Wang, Z. Neutrophil-Mediated Delivery of Therapeutic Nanoparticles across Blood Vessel Barrier for Treatment of Inflammation and Infection. ACS Nano 2015, 9 (12), 11800-11811. 
(20) Wang, Q.; Kuo, Y.; Wang, Y.; Shin, G.; Ruengruglikit, C.; Huang, Q. Luminescent Properties of Water-Soluble Denatured Bovine Serum Albumin-Coated CdTe Quantum Dots. J. Phys. Chem. B 2006, 110, 16860-16866.

(21) Shao, L.; Dong, C.; Sang, F.; Qian, H.; Ren, J. Studies on Interaction of CdTe Quantum Dots with Bovine Serum Albumin Using Fluorescence Correlation Spectroscopy. J Fluoresc 2009, 19,151-157.

(22) Liang, J.; Cheng, Y.; Han, H. Study on the Interaction between Bovine Serum Albumin and CdTe Quantum Dots with Spectroscopic Techniques. J. Mol. Struct. 2008, 892, 116-120.

(23) Wang, Q.; Zhang, X.; Zhou, X.; Fang, T.; Liu, P.; Liu, P.; Min, X.; Li, X. Interaction of Different Thiol-Capped CdTe Quantum Dots with Bovine Serum Albumin. J. Lumin. 2012, 132, $1695-1700$.

(24) Idowu, M.; Lamprecht, E.; Nyokong, T. Interaction of Water-Soluble Thiol Capped CdTe Quantum Dots and Bovine Serum Albumin. J. Photochem. Photobiol., A 2008, 198, 7-12.

(25) Jhonsi, M.A.; Kathiravan, A.; Renganathan, R. Spectroscopic Studies on the Interaction of Colloidal Capped CdS Nanoparticles with Bovine Serum Albumin. Colloids Surf., B 2009, 72, $167-172$

(26) Dzagli, M.M.; Canpean, V.; Iosin, M.; Mohou, M.A.; Astilean, S. Study of the Interaction between CdSe/ZnS Core-Shell Quantum Dots and Bovine Serum Albumin by Spectroscopic Techniques. J. Photochem. Photobiol., A 2010, 215, 118-122. 
(27) Zhang, B.; Wang, X.; Liu, F.; Chengand, Y.; Shi, D. Effective Reduction of Nonspecific Binding by Surface Engineering of Quantum Dots with Bovine Serum Albumin for CellTargeted Imaging. Langmuir 2012, 28 (48), 16605-16613.

(28) Nida, D.L.; Nitin, N; Yu, W.W.; Colvin, V.L.; Richards-Kortum R. Photostability of Quantum Dots with Amphiphilic Polymer-Based Passivation Strategies. Nanotechnology 2008, 19(3), 035701.

(29) Ma, Q.F.; Chen, J.Y.; Wu, X.; Wang, P.N.; Yue, Y.; Dai, N. Photostability Comparison of CdTe and CdSe/CdS/ZnS Quantum Dots in Living Cells under Single and Two-Photon Excitations. J. Lumin. 2011, 131, 2267-2272.

(30) Tsipotan, A.S.; Gerasimova, M.A.; Aleksandrovsky, A.S.; Zharkov, S.M.; Slabko V.V. Effect of Visible and UV Irradiation on the Aggregation Stability of CdTe Quantum Dots. $J$ Nanopart Res 2016, 18, 324.

(31) Frame, F.A.; Carroll, E.C.; Larsen, D.S.; Sarahan, M.; Browning, N.D.; Osterloh, F.E. First Demonstration of CdSe as a Photocatalyst for Hydrogen Evolution from Water under UV and Visible Light. Chem. Commun. 2008, 2206-2208.

(32) Zhao, J.; Holmes, M.A.; Osterloh, F.E. Quantum Confinement Controls Photocatalysis: A Free Energy Analysis for Photocatalytic Proton Reduction at CdSe Nanocrystals. ACS Nano 2013, 7 (5), 4316-4325.

(33) Krauss, T.D.; Peterson, J.J.; A Charge for Blinking. Nature Materials 2012, 11, 14-17. 
(34) Pechstedt, K.; Whittle, T.; Baumberg, J.; Melvin, T. Photoluminescence of Colloidal CdSe/ZnS Quantum Dots: The Critical Effect of Water Molecules. J. Phys. Chem. C 2010, 114, 12069-12077.

(35) Emin, S.; Loukanov, A.; Wakasa, M.; Nakabayashi, S.; and Kaneko, Y. Photostability of Water-dispersible CdTe Quantum Dots: Capping Ligands and Oxygen. Chem. Lett. 2010, 39, 654-656.

(36) Gulnov, D.V.; Nemtseva, E.V.; Kratasyuk, V.A. Contrasting Relationship between Macro- and Microviscosity of the Gelatin- and Starch-Based Suspensions and Gels. Polym. Bull. 2016, 73 (12), 3421-3435.

(37) Comorosan. S.; Polosan, S.; Popescu, I.; Ionescu, E.; Mitrica, R.; Cristache, L.; State, A.E. Structural Studies on Serum Albumins under Green Light Irradiation. Eur Biophys $J$ 2010, 39, $1483-1491$.

(38) Boldt, K.; Bruns, O.T.; Gaponik, N.; Eychmüller, A. Comparative Examination of the Stability of Semiconductor Quantum Dots in Various Biochemical Buffers. J. Phys. Chem. B 2006, $110(5), 1959-1963$.

(39) Karpov, S.V.; Gerasimov, V.S.; Grachev, A.S.; Isaev, I.L.; Podavalova, O.P.; Slabko, V.V. Experimental Manifestations of the Correlation between the Local Structure of Silver Nanoparticle Aggregates and their Absorption Spectra. Colloid J. 2007, 69, 170-179.

(40) Yang, P.; Ando, M.; Murase, N. Highly Luminescent CdSe/Cd $\mathrm{Zn}_{1-\mathrm{x}} \mathrm{S}$ Quantum Dots Coated with Thickness-Controlled SiO2Shell through Silanization. Langmuir 2011, 27, 95359540. 
(41) Ibrahim, S.A.; Ahmed, W.; Youssef, T. Photoluminescence and Photostability Investigations of Biocompatible Semiconductor Nanocrystals Coated with Glutathione Using Low Laser Power. J Nanopart Res 2014, 16, 2445. 
TOC Graphic

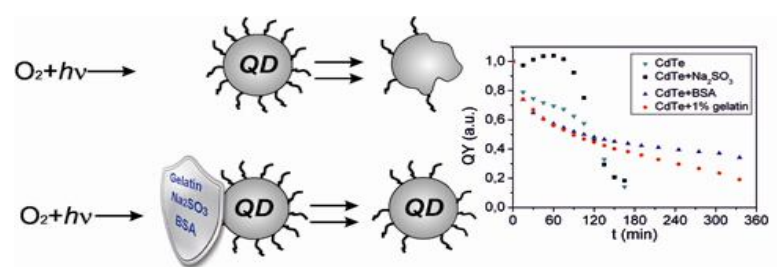

